\title{
Molecular dynamics simulations of the contact angle between water droplets and graphite surfaces
}

\author{
Danilo Sergi, Giulio Scocchi, and Alberto Ortona \\ University of Applied Sciences (SUPSI), The iCIMSI Research Institute, Galleria 2, CH-6928 Manno, Switzerland
}

(Dated: August 28, 2012)

\begin{abstract}
Wetting is a widespread phenomenon, most prominent in a number of cases, both in nature and technology. Droplets of pure water with initial radius ranging from 20 to $80[\AA]$ spreading on graphitic surfaces are studied by molecular dynamics simulations. The equilibrium contact angle is determined and the transition to the macroscopic limit is discussed using Young equation in its modified form. While the largest droplets are almost perfectly spherical, the profiles of the smallest ones are no more properly described by a circle. For the sake of accuracy, we employ a more general fitting procedure based on local averages. Furthermore, our results reveal that there is a possible transition to the macroscopic limit. The modified Young equation is particularly precise for characteristic lengths (radii and contact-line curvatures) around $40[\AA]$.
\end{abstract}

Keywords: wetting;contact angle;graphite;molecular dynamics simulations

\section{INTRODUCTION}

Wettability is a long-standing issue primarily addressed by looking at the angle of contact at the edge of the interface between a liquid and a solid (sessile droplet method) [1]. In spite of the simplicity of the formulation of the problem, this procedure is at the basis of many investigations devised to assess the behavior of a liquid or a material in a number of industrial processes and applications (see Ref. [2] for a review), and it has been demanding valuable efforts, both experimental and theoretical [3-6]. Computer simulations provide useful guidelines for their power to deal with the complexity of large assemblies of interacting components. In classical molecular dynamics studies, the systems are described at the molecular level according to the laws of classical mechanics and electrodynamics. For these reasons, this approach proves to be both versatile and accurate. Recent breakthroughs have triggered a burst of interest in the properties of graphene [7-9]. Promising applications in a variety of fields could indeed develop. Our interest in graphitic materials is related to their optimal dispersion in polymeric matrices for the best manufacturing of composite materials [10-12]. In that respect, the wetting properties of graphitic surfaces by water are of course the starting point of any subsequent investigation. In particular, the transition to the macroscopic limit is essential for any further study adopting more advanced modelization schemes [13, 14]. Our analysis of profiles differs from the well-established method [15, 16] in that we approximate them by a more general curve than a circle. This way of proceeding is especially necessary for small droplets, exhibiting the largest deviations from the predictions of Young equation and from a spherical cap. This last aspect has already been recognized in previous studies [17]. Yet, we also propose an analytic expression for the oxygen-oxygen radial distribution function of water.

\section{SIMULATIONS}

All molecular dynamics simulations are performed with LAMMPS [18], a code that supports parallelization optimally [19]. Numerical integration is accomplished with the algorithm rRESPA, allowing to deal with multiple time step sizes
[18]. We choose a time step of 2 [fs] for non-bonded interactions and of 1 [fs] for bonded interactions. All Lennard-Jones forces are evaluated with a potential of the type 12-6. The inbuilt CHARMM force field [20] is employed to prevent van der Waals interactions from decaying abruptly at the cutoff distance: the forces are smoothly corrected to zero from 10 to 12 [Å]. Pairwise Coulomb interactions within a distance of $12[\AA]$ are calculated in the real space and beyond this value with a particle-particle, particle-mesh method; the precision is set to $10^{-4}$. The pairwise interactions among atoms separated by one or more bonds are neglected. The neighbor lists are always updated at every time step. These general settings are always applied, unless specified otherwise.

\section{A. Water}

Throughout our work we use for the water the $\mathrm{SPC} / \mathrm{Fw}$ model introduced in Ref. [21]. We address the reader to this detailed study for the definitions (partial charges, equilibrium distances, interaction parameters, etc.). We start from 512 molecules of water arranged regularly in a cubic box of side length $30[\AA]$ with periodic boundary conditions. We let the system evolve for 200 [ps] in the ensemble NPT (NoséHoover integration). This simulation is performed as equilibration with a single time step size of 1 [fs]. The target temperature and pressure are $298.16[\mathrm{~K}]$ and 1 [atm], respectively. Since the main purpose is to reproduce experimental densities, we choose the parameters that control the convergence so as to fix the temperature and the volume, while we still let the pressure fluctuate. It is in fact well-known that the pressure is a very sensitive function of the volume and difficult to equilibrate accurately [22]. We then let the system evolve for other 0.5 [ns] at NVE conditions; the final configuration of this dynamics is replicated and used to obtain the droplets of water.

\section{B. Droplets and graphitic substrate}

All starting configurations are formed by two parallel planes of graphene and a semisphere of molecules of water. The planes of graphene are separated by $3.4[\AA]$ with 
the lower one translated by the vector $(l / 2, \sqrt{3} l / 2)$ with respect to the first; $l=1.42[\AA]$ is the length of the bonds among the carbon atoms. The two planes are approximately squared with side of at least $30[\AA]$ larger than the diameter of the semisphere. The semisphere is centered above the planes of graphene at a distance of $3[\AA]$ from the upper plane. The boundaries are periodic and two images of the droplets are separated by at least $100[\AA]$ in the $z$ direction. The mass of the carbon atoms is $m_{\mathrm{C}}=12.011[\mathrm{~g} / \mathrm{mol}]$. The water and the graphene interact via van der Waals forces between the atoms of carbon and oxygen with force field parameters $\varepsilon_{\mathrm{CO}}=0.0478[\mathrm{kcal} / \mathrm{mol}]$ and $\sigma_{\mathrm{CO}}=3.581[\AA][23,24]$. The system is equilibrated for 0.5 [ns] in the ensemble NVT (Nosé-Hoover thermostat) with the temperature of the water maintained at 298.16 [K]. The system is studied during a further evolution of 1 [ns] in the microcanonical ensemble by gathering frames at every 0.5 [ps].

\section{ANALYSIS}

\section{A. Water}

The density of water is calculated using the formula $\rho=$ $N\left(m_{\mathrm{O}}+2 m_{\mathrm{H}}\right) /\left(3 \cdot 0.602 \cdot V_{\mathrm{dom}}\right) . \quad N$ is the total number of atoms and $V_{\text {dom }}$ is the volume in $\AA^{3}$ of the cubic domain resulting from the preliminary simulation of equilibration. By using this formula the result is expressed in $\mathrm{g} / \mathrm{cm}^{3}$. The oxygen-oxygen radial distribution function $g(r)$ is defined by $\left(N / 3 V_{\text {dom }}\right) g(r) 4 \pi r^{2} \Delta r=W(r) . W(r)$ is the average number of oxygen atoms in a shell of width $\Delta r$ at a distance $r$ from a given oxygen atom. We choose $\Delta r=0.05[\AA]$ and of course $g(r)$ is unitless. We also calculate the cumulative probability $P(r)=(3 / N) \int_{0}^{r} W(r) n \mathrm{~d} r$, which yields the fraction of oxygen atoms within a distance $r$ from a given atom of oxygen. $n=1 / \Delta r$ is the number of shells per unit length. (By writing the above integral as a discrete sum, that sum would run over the number of shells.)

\section{B. Droplets}

The contact angle $\theta$ is defined by the tangent at the contact line, the edge of the interface between the solid and liquid phases (see Fig. 3). The contact angle of a macroscopic droplet with spherical symmetry is well described by the equation $\cos \theta=\left(\gamma_{\mathrm{sv}}-\gamma_{\mathrm{sl}}\right) / \gamma_{\mathrm{lv}}$. The $\gamma^{\prime}$ s are the surface/interfacial tensions. The subscripts s, 1 and $\mathrm{v}$ stand for solid, liquid and vapor, respectively. The above relation is generally referred to as Young equation [1]. Especially for small droplets, Young equation needs a corrective term that accounts for the line tension, leading to $\cos \theta=\cos \theta_{\infty}-\kappa /\left(\gamma_{\mathrm{v}} R\right)$. As the notation suggests, $\cos \theta_{\infty}=\left(\gamma_{\mathrm{sv}}-\gamma_{\mathrm{sl}}\right) / \gamma_{\mathrm{lv}}$ is Young equation, which yields the contact angle $\theta_{\infty}$ in the macroscopic limit. $R$ is the radius (or curvature) of the contact line and $\kappa$ is the line tension. If $\theta<90^{\circ}$, we speak about hydrophilic behavior; hydrophobic if $\theta>90^{\circ}$. Complete wetting corresponds to $\theta=0^{\circ}$. The discussion of the contact angle is

\begin{tabular}{lccc}
\hline \hline$L_{\text {dom }}[\AA]$ & $V_{\text {dom }}\left[\AA^{3}\right]$ & $\rho\left[\mathrm{g} / \AA^{3}\right]$ & $d\left[\right.$ molecules $\left./ \AA^{3}\right]$ \\
\hline 24.835 & $15^{\prime} 318$ & 1.0002 & 0.0334 \\
\hline \hline
\end{tabular}

Table I: Length of the side of the cubic simulation domain resulting from equilibration, its volume, mass and molecular densities.

much richer than what reported here. A more detailed treatment can be found in Refs. [2-5]. In order to obtain the profile of the droplets, it is applied the method explained in Refs. [15, 16, 24] with $\Delta V=1.9 \times 10^{-4}\left[\AA^{3}\right]$. Typically, the contact angle is extracted by superimposing a circle on the profile coming out from the simulations. The center and the radius of the circle are obtained by a fit. Here we have decided to proceed in a quite different way. Indeed, there appears that the circle departs from the shape of the droplets in particular for the smallest ones where the contact angle is calculated, because its radius of curvature is weaker. We thus approximate the profile of the droplets by a piecewise linear function. The idea is to subdivide the profile into small elements. For the points falling within an element, we calculate the average values for both $x$ and $y$ coordinates. These average values are the usual parameters used in linear regressions. The contact angle is calculated from the slope of the linear function obtained by a linear regression on the average points above the contact line at most $5[\AA]$. The contact line point is assumed to be at $y=2^{1 / 6} \sigma_{\mathrm{CO}}$. The contact area is simply given by $C=\pi R^{2}$; the contact line is $L=2 \pi R$. The overall interfacial surface of the droplets is calculated by means of the formula $S=2 \pi r^{2}+2 \pi r\left(r+\sqrt{r^{2}-R^{2}}\right)$ and their volume with $V=(2 / 3) \pi r^{3}+\pi r^{2} \sqrt{r^{2}-R^{2}}-(\pi / 3)\left(r^{2}-R^{2}\right)^{3 / 2}$.

\section{RESULTS AND DISCUSSION}

\section{A. Water}

Table I] summarizes some final results for the box of $\mathrm{SPC} / \mathrm{Fw}$ water that is used as source in order to extract the droplets. Our findings are in good agreement with the original work for that atomistic water model [21]. Figure 1 shows the oxygen-oxygen radial distribution function. The results for the cumulative probability $P(r)$ in Fig. 2 indicate that the behavior of water differs slightly from that of a continuum, homogeneous medium, except in the closest neighborhood of the oxygen atoms. The inset tells us that technically the first peak of the radial distribution function is related to the derivative of a step function (or Heaviside function). We thus consider a function of the type $f(r)=(4 / 3)\left(\pi r^{3} / L_{\text {dom }}^{3}\right) /\left(1+\mathrm{e}^{(A-r) / B}\right)$. Of course, this function is no more normalized to unity in the interval $\left[0, L_{\mathrm{dom}}\right)$, but it provides a good approximation of the cumulative distribution $P(r)$ where the first peak of the radial distribution function occurs. The denominator of $f(r)$ is reminiscent of the Fermi-Dirac distribution function, which is a step function at low temperatures. The parameter $A$ fixes the position of the first peak and the parameter $B$ determines its width and height. After a simple calculation, we find for the 


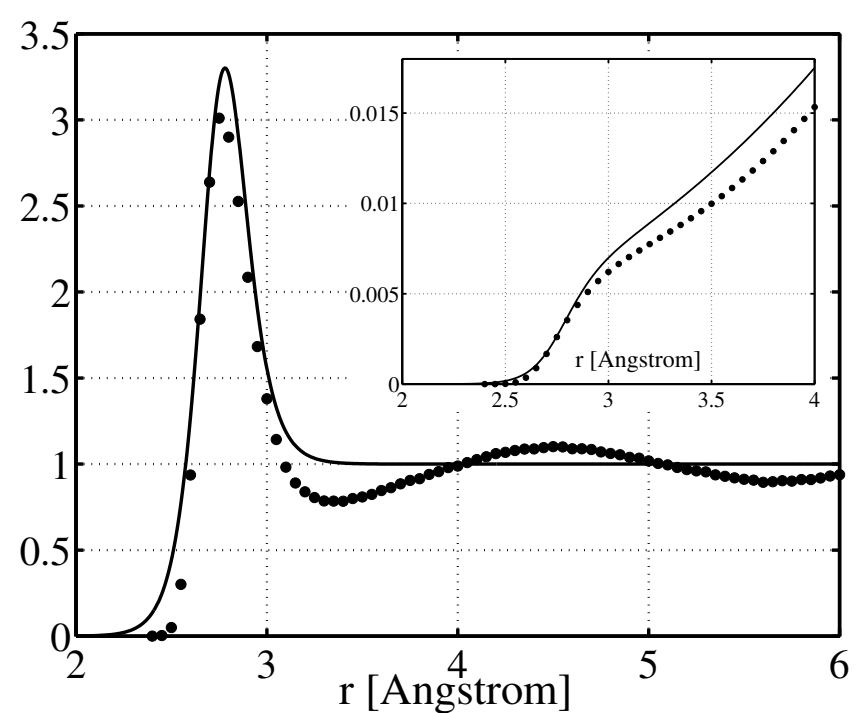

Figure 1: Oxygen-oxygen radial distribution function of water. The solid line is the plot of the function $u(r)$, Eq. 11 The parameters $A$ and $B$ are the result of a fit to the data, represented as filled circles. We find $A=2.7610 \pm 0.0329[\AA]$ and $B=0.0833 \pm 0.0183[\AA]$. Inset: Plot of the function $f(r)$ (see main text), with the same parameters $A$ and $B$ (solid line) compared to the same data of Fig.2(filled circles). The two curves differ at most of $2.25 \cdot 10^{-3}$.

radial distribution function the following analytic expression:

$$
u(r)=\frac{1}{1+\mathrm{e}^{(A-r) / B}}\left[1+\frac{r}{3 B} \frac{\mathrm{e}^{(A-r) / B}}{1+\mathrm{e}^{(A-r) / B}}\right] .
$$

This function reproduces correctly the first peak, as shown in Fig. 1. The main discrepancy with the data for the SPC/Fw model is in the neighborhood of the first minimum, corresponding to a density depletion with respect to the bulk value. The above method is of course unable to explain this aspect of the fine structure of water.

\section{B. Droplets}

Figure 3 shows the profile of a small droplet when approximated by a piecewise linear function. Near the contact line, it appears that this procedure is more precise than a circular fit. From local averages, the resulting contact angle is always lower (see Fig. 47. In both cases, the macroscopic contact angle can be extracted from an average over the largest droplets, those of radii $60-80[\AA]$, leading to $\theta_{\infty}=103.1^{\circ}$ $\left(\theta_{\infty}=108.1^{\circ}\right.$ for circular fits). Its standard deviation is $0.9 \%$ $(0.3 \%)$ of this average value. The residual difference between the two methods indicates that, even for the largest droplets, in the close neighborhood of the contact line, the profile still deviates from a perfectly spherical cap. It turns out that the method based on circular fits underestimates the base radius. The average bulk molecular density is 0.0323 [molecules $/ \AA^{3}$ ] (cf. Tab. I), with standard deviation $3.5 \%$ of it. By fitting the data to the modified Young equation it is extrapolated a value of $\theta_{\infty}=111.1^{\circ}\left(110.7^{\circ}\right.$ for circular fits). Especially

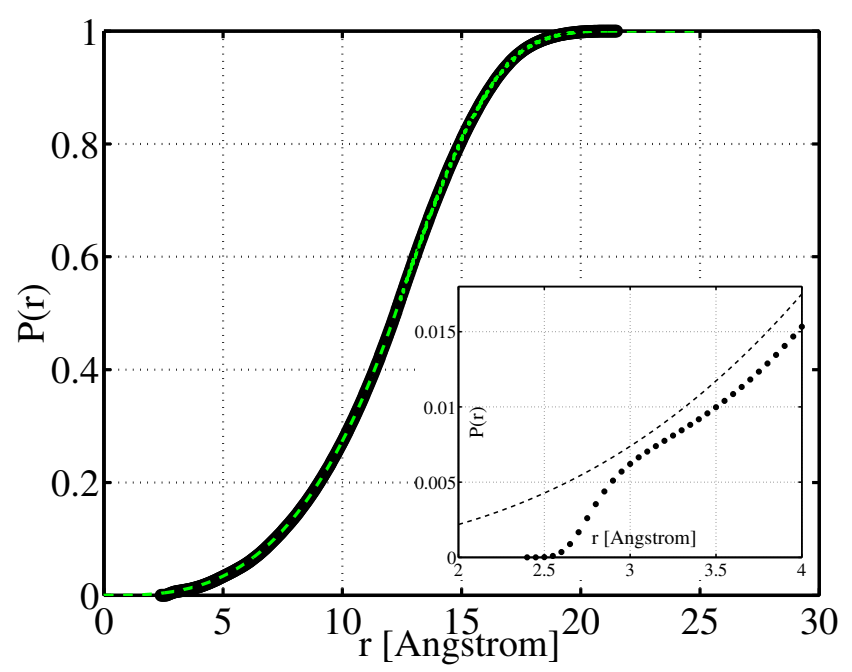

Figure 2: Cumulative probability $P(r)$ (see main text). The dashed line accounts for the continuum, homogeneous behavior. We start from a sphere centered at the origin. When the radius of the sphere is larger than $L_{\mathrm{dom}} / 2$ (see Tab. I), the sphere overlaps with itself because of periodic boundary conditions and we can no more use the formula $P(r)=(4 / 3) \pi r^{3} / L_{\mathrm{dom}}^{3}$. We thus employ the Monte Carlo principle in its elementary form. For a given radius larger than $L_{\mathrm{dom}} / 2,30^{\prime} 000$ points are randomly (uniform distribution) placed in a cube of side $L_{\mathrm{dom}}$ and we count the fraction of them falling within the portions of the sphere. Inset: Magnification around the position of the first peak of the radial distribution function (cf. Fig. 1).

for the circular profiles, we prefer the first method because the results indicate that around $60[\AA]$ occurs a transition to the macroscopic limit (see Inset of Fig. 4 and previous basic statistics). From a typical value for the surface tension of water of $\gamma=72[\mathrm{mN} / \mathrm{m}]$, for the line tension it is found $\kappa=-2.04 \cdot 10^{-11}[\mathrm{~N}]$ from circular fits and $\kappa=-5.35 \cdot 10^{-11}$ $[\mathrm{N}]$ from local averages. This means that the forces tending to expand the contact area are higher according to the method based on local averages. In Fig. 5 we compare the contact area to interfacial area ratio, i.e. $C / S$, with the results for the droplets having the same final radius but with contact angle $\theta_{\infty}=108.1^{\circ}$ (the value of $110.7^{\circ}$ underestimates significantly $C / S$ for the largest droplets). Given a final radius, the curvature of the contact line is determined numerically so as to have the macroscopic contact angle $\theta_{\infty}$. With respect to the macroscopic expectation, it is found that for the five smaller droplets on average the contact area expands of $4.2 \%$, the interfacial surface shrinks of $2.8 \%$ and the volume contracts of $7.2 \%$. Figure 6 compares the density maps of three droplets of different size. These representations suggest that, for small droplets, a higher fraction of molecules is involved in density fluctuations at the interface. A simple calculation shows that this aspect effectively occurs if $N_{1} / N_{2}>\rho_{1} / \rho_{2}$ holds. The index 1 designates a large droplet and 2 a smaller one. $N$ is the number of atoms in the fluid phase and $\rho$ the bulk density. If we compare the droplets of initial radii 30 and 60 [ $\AA$, we find $\left(3 \cdot 15^{\prime} 119\right) /\left(3 \cdot 1^{\prime} 902\right)>0.0338 / 0.0337=1$. Since even for the smallest droplet of initial radius of $20[\AA \AA]$ the den- 


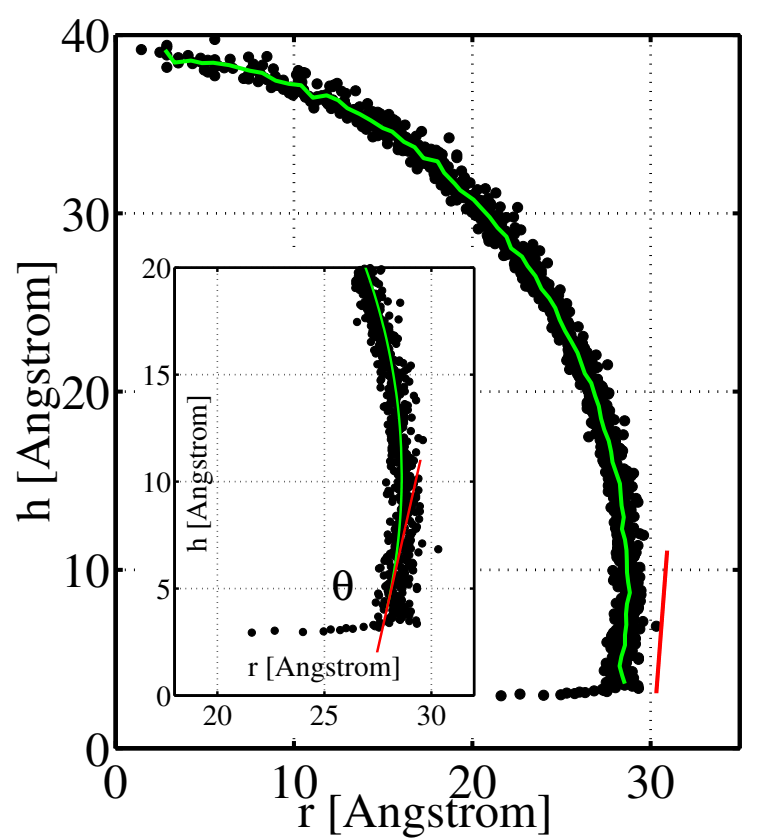

Figure 3: Profile of the droplet of initial radius of 30 [Å]. The green curve represents the profile of the droplet as obtained from local averages. The straight line is a guide for the eyes and its slope amounts to the value used for determining the contact angle. Inset: Profile from a circular fit to the data in the neighborhood of contact line. The straight line is the tangent at the contact-line point.

sity of water is close to the bulk value and the surface thickness is around $3[\AA]$, we conclude that for sure larger droplets have a reduced fraction of molecules at the interface. In other words, as the droplet increases in size, its interface grows and the fraction of molecules fluctuating at the interface becomes smaller. The droplet of initial radius $60[\AA]$ was also simulated at different temperatures up to $450[\mathrm{~K}]$. For this temperature increase, it is found that the contact angle varies with good approximation linearly, as well as the molecular density. In contrast to what reported in Ref. [25] the contact angle varies over this temperature range only of a few degrees. Preliminary results using coarse-grained models confirm our trend.

\section{CONCLUSIONS}

Contact angle measurements and comparisons with the predictions of Young equation are generally carried out under the assumption of a spherical shape of droplets. Figure 5 shows that, when the contribution of the corrective term to Young equation and/or other size effects [6, 17, 24] is more important, the droplets deviate significantly from the macroscopic expectation. Furthermore, below the initial radius of $30[\AA]$, the contact angle can no more be derived accurately from the tangent to a circular profile at the contact line (see Figs. 3 and 44. Actually, from our analysis it clearly emerges that, for small droplets, fluctuations of the surface thickness are of major relevance, resulting in the deformation of their spherical shape. For small droplets the effect is more marked presum-

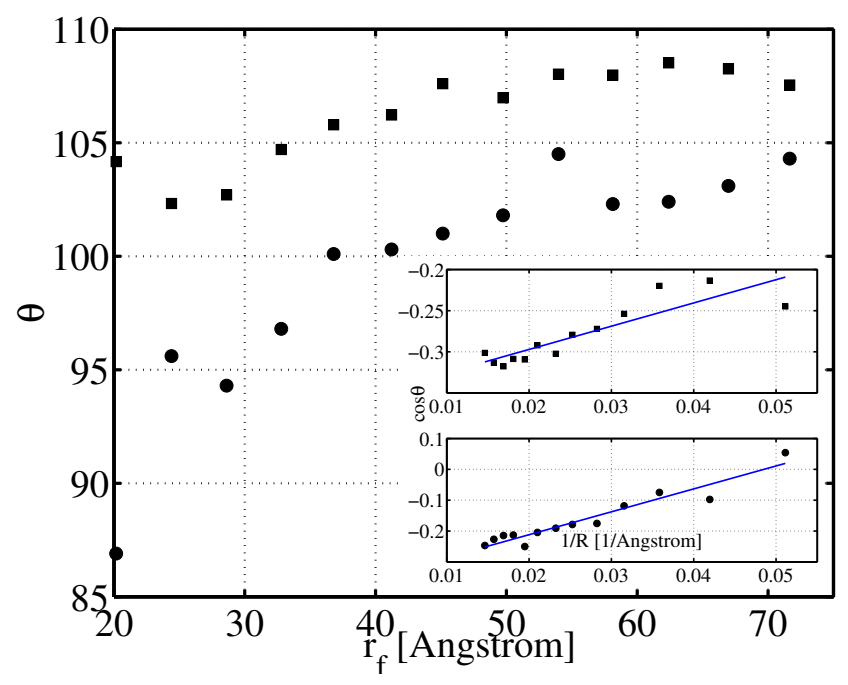

Figure 4: Contact angle dependence on the final radius from the two approximations of the droplet profiles (squares for circular fits and circles for local averages). Inset: Fitting of the data to modified Young equation with contact angles resulting from circular fits, Top, and from local averages, Bottom.

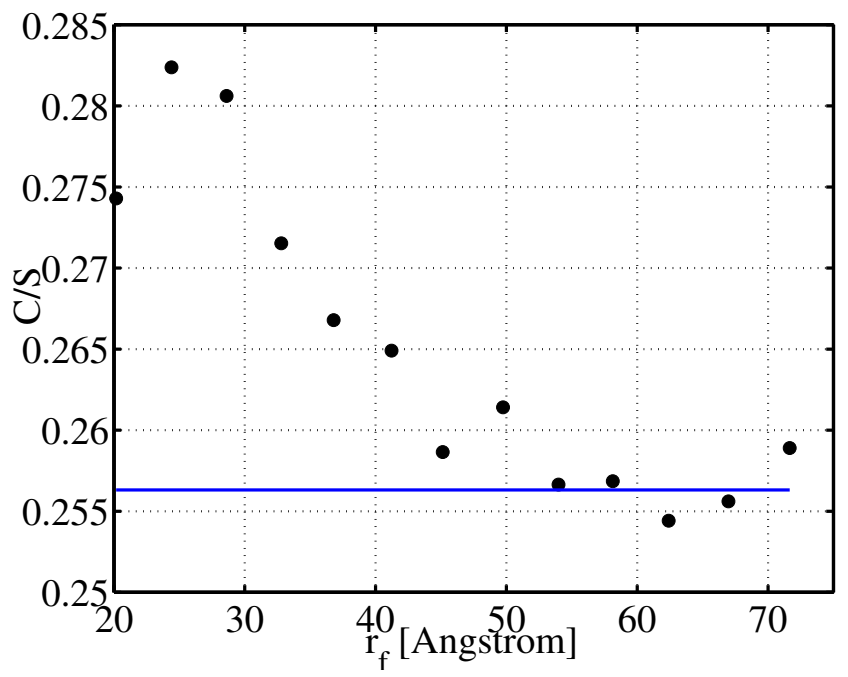

Figure 5: Contact area to overall interfacial surface ratio as a function of the final radius of the droplets. The straight line is the macroscopic expectation under the assumption of a perfectly spherical profile.

ably because of their reduced size and the short-range nature of non-bonded interactions (cf. Ref. [24]): cohesive forces near the contact line are weaker and the contact area would tend to expand, leading to lower contact angles. Interestingly, it also appears that the macroscopic limit is not reached gradually, but with a possible transition around the initial radius of $60[\AA]$. On the other hand, the predictions of the modified Young equation are more precisely recovered for initial radii around $40[\AA]$. Finally, the SPC/Fw model for water is extensively validated [21] and the Lennard-Jones parameter $\varepsilon_{\mathrm{CO}}$ used here resulted from a previous calibration accord- 

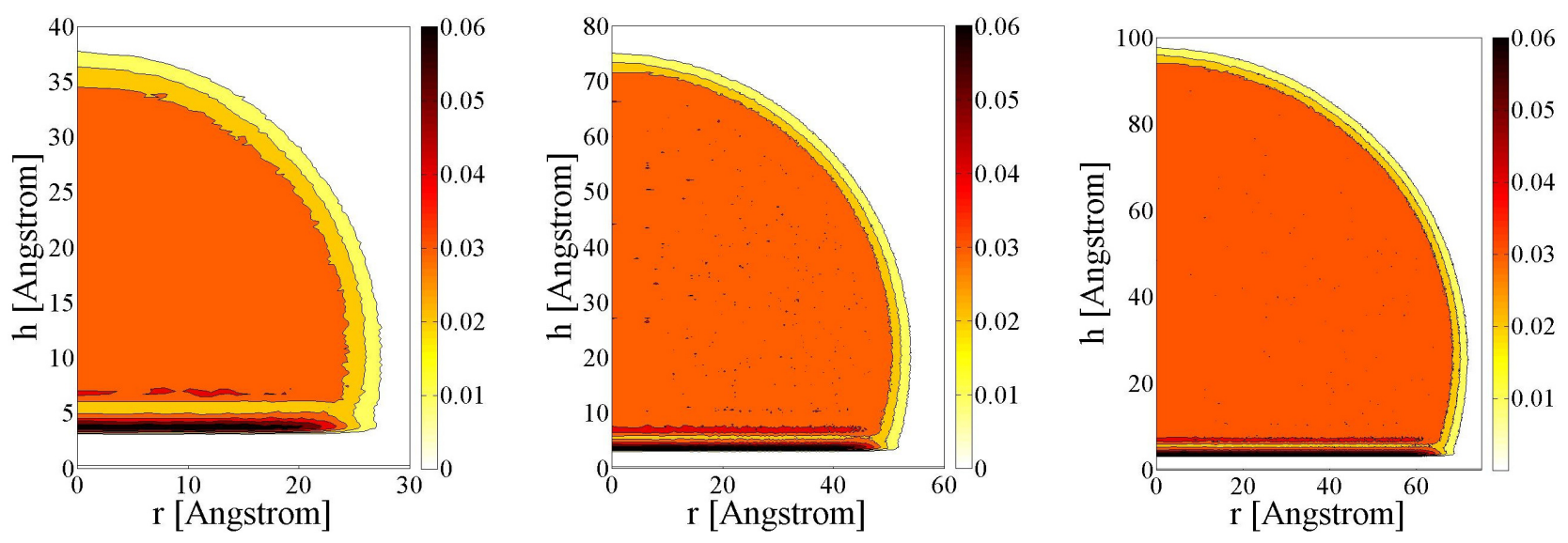

Figure 6: Density maps for the droplets of initial radii 30, 60 and $80[\AA]$, in this order. Color code based on molecular density. The thickness of the interface is in all cases around $3[\AA]$. Concerning the first map, the horizontal shift relative to the representation of Fig. 3 is due to the fact that the first radial bin coincides with zero and the coarser binning necessary for this kind of representation.

ing to recent measurements carried out under the most ideal conditions [23, 24]. Our macroscopic contact angle $\theta_{\infty}$ of $111.1^{\circ}$ (circular fits and extrapolated value) underestimates the experimental value of $127^{\circ}$ [23], taken as reference for calibration [24]. We ascribe this discrepancy mostly to the different cutoff scheme and the inclusion of long-range interactions [26]. Clearly, our analysis of profiles and the main conclusions drawn for the smallest droplets in the hydrophobic regime are robust under small changes of the simulation settings. Indeed, the profiles of these droplets will be affected to a larger extent by the fluctuations of the surface thickness and in turn no more fully spherical. The accuracy implied by the various simulation settings confer a substantial degree of confidence to the findings reported here.

\section{NOMENCLATURE}

$\begin{array}{ll}A, B & \AA \\ C & \AA^{2} \\ d & \AA^{-3} \\ f, u & - \\ g & - \\ L & \AA \\ L_{\mathrm{dom}} & \AA \\ l & \AA \\ m & \mathrm{~g} \cdot \mathrm{mol}^{-1} \\ N & - \\ n & \AA \\ & \\ P & -\end{array}$

contact area of droplets

molecular density

model functions

radial distribution function

length of contact line of droplets

side length of simulation domain

carbon bond length in graphene

mass of atoms

number of atoms

number of spherical shells per unit length

cumulative radial distribution for oxy-

$\begin{array}{ll}R & \AA \\ r & \AA \\ \Delta r & \AA \\ S & \AA^{2} \\ V & \AA^{3} \\ V_{\mathrm{dom}} & \AA^{3} \\ W & - \\ x, y, h & \AA \\ \varepsilon & \mathrm{kcal} \cdot \mathrm{mol}^{-1} \\ \gamma & \mathrm{N} \cdot \mathrm{m}^{-1} \\ \kappa & \mathrm{N} \\ \sigma & \AA \\ \rho & \mathrm{g} \cdot \mathrm{cm}^{-3} \\ \theta & -\end{array}$
gen atoms

\author{
base radius of droplets \\ radius of droplets \\ width of a spherical shell \\ overall interfacial surface of droplets \\ volume of droplets \\ volume of simulation domain \\ average number of oxygen atoms in a \\ spherical shell \\ cartesian coordinates \\ interaction parameter for Lennard-Jones \\ potential \\ surface/interfacial tension \\ line tension \\ interaction parameter for Lennard-Jones \\ potential \\ mass density \\ contact angle
}

\section{Acknowledgments}

This is work supported by the Swiss Innovation Promotion Agency (KTI/CTI) under grant P. No. 10055.1 (BiPCaNP project). Computations were done with the facilities of CSCS and iCIMSI-SUPSI. We thank their staff for assistance. We are also grateful to the anonymous Referees for their comments on a previous version of this work. 
Vol. 6, Koninklijke Brill NV, Leiden, 2009.

[3] A. Méndez-Vilas, A.B. Jódar-Reyes, M.L. González-Martín, small 5 (2009) 1366-1390.

[4] D. Quéré, Annu. Rev. Mater. Res. 38 (2008) 71-99.

[5] P.G. de Gennes, Rev. Mod. Phys. 57 (1985) 827-863.

[6] L. Schimmele, M. Napiórkowski, S. Dietrich, J. Chem. Phys. 127 (2007) 164715-164742.

[7] H.W. Kroto, J.R. Heath, S.C. O'Brien, R.F. Curl, R.E. Smalley, Nature 318 (1985) 162-163.

[8] S. Iijima, Nature 354 (1991) 56-58.

[9] K.S. Novoselov, A.K. Geim, S.V. Morozov, D. Jiang, Y. Zhang, S.V. Dubonos, I.V. Grigorieva, A.A. Firsov, Science 306 (2004) 666-669.

[10] M.F. Islam, E. Rojas, D.M. Bergey, A.T. Johnson, A.G. Yodh, Nano Lett. 3 (2003) 269-273.

[11] L. Vaisman, H.D. Wagner, G. Marom, Adv. Colloid Interface Sci. 128 (2006) 37-46.

[12] E.T. Thostenson, C. Li, T.-W. Chou, Compos. Sci. Technol. 65 (2005) 491-516.

[13] W. Shinoda, R. DeVane, M.L. Klein, Mol. Sim. 33 (2007) 2736.

[14] S.J. Marrink, H.J. Risselada, S. Yefimov, D.P. Tieleman, A.H. de Vries, J. Phys. Chem. B 111 (2007) 7812-7824.

[15] M.J. de Reijter, T.D. Blake, J. De Coninck, Langmuir 15 (1999) 7836-7847.

[16] T. Werder, J.H. Walther, R.L. Jaffe, T. Halicioglu, P. Koumoutsakos, J. Phys. Chem. B 107 (2003) 1345-1352.
[17] T. Ingebrigtsen and S. Toxvaerd, J. Phys. Chem. C 111 (2007) 8518-8523.

[18] S.J. Plimpton, R. Pollock, M. Stevens, in: Proc. of Eighth SIAM Conf. on Parallel Processing for Scientific Computing, Minneapolis, 1997; Available at http://www.cs.sandia. gov/s jplimp/lammps.html.

[19] S. Plimpton, J. Comp. Phys. 117 (1995) 1-19.

[20] A.D. MacKerell Jr, D. Bashford, M. Bellott, R.L. Dunbrack Jr, J.D. Evanseck, M.J. Field, S. Fisher, J. Gao, H. Guo, S. Ha, D. Joseph-McCarthy, L. Kuchnir, K. Kuczera, F.T.K. Lau, C. Mattos, S. Michnick, T. Ngo, D.T. Nguyen, B. Prodhom, W.E. Reiher III, B. Roux, M. Schlenkrich, J.C. Smith, R. Stote, J. Straub, M. Watanabe, J. Wiorkiewick-Kuczera, D. Yin, M. Karplus, J. Phys. Chem. 102 (1998) 3586-3616.

[21] Y. Wu, H.L. Tepper, G.A. Voth, J. Chem. Phys. 124 (2006) 24503-24514.

[22] LAMMPS Documentation, http://lammps.sandia. gov/doc/Manual.html

[23] S. Wang, Y. Zhang, N. Abidi, S. Cabrales, Langmuir 25 (2009) 11078-11081.

[24] G. Scocchi, D. Sergi, C. D’Angelo, A. Ortona, Phys. Rev. E. 84 (2011) 61602-61609.

[25] R.C. Dutta, S. Khan, J.K. Singh, Fluid Phase Equilibria 302 (2011) 310-315.

[26] R.L. Jaffe, P. Gonnet, T. Werder, J.H. Haliciogu, P. Koumoutsakos, Mol. Sim. 30 (2003) 205-216. 